\title{
Awareness of orthodontic treatment need in young adults between 18-25 years
}

\author{
Anirudh K Mathur', Swaroopa Ponnada ${ }^{2, *}$, NKS Aravind ${ }^{3}$, T.V. Pavan ${ }^{4}, \operatorname{Prasad~Chitra~}^{5}$ \\ ${ }^{1,3}$ Reader, Dept. of Orthodontics, Army College of Dental Sciences, Telangana, ${ }^{2}$ Reader, Dept. of Orthodontics, Panineeya Dental \\ College, Telangana, ${ }^{4}$ PG Student, ${ }^{5}$ Professor and Head, Dept. of Orthodontics, Army College of Dental Sciences, Telangana, \\ India
}

*Corresponding Author:

Email: swaroopaponnada@gmail.com

\begin{abstract}
Introduction: Health is defined by WHO as 'a state of complete physical, mental, and social well-being and not merely the absence of disease or infirmity.

Objectives: There is a need to identify levels of awareness with respect to Orthodontic treatment need in the adult group of patients who comprise the majority of orthodontic patients.

Materials and Methods: A survey of 14 questionnaire was conducted among the students of various colleges in Hyderabad and Secunderabad, India. A total of 808 young adult patients of age group of 18-25 years were included in the study.

Data Analysis: Chi-square test, unpaired t test and one way ANOVA were performed.

Results and Discussion: Most of the students participated in the survey responded that they noticed people with crooked teeth and had seen people wearing braces $(76.73 \%$ and $83.91 \%$ respectively). However, when asked the need for wearing braces, most of them responded as not required $(74.13 \%)$. Around $52 \%$ felt that the orthodontic treatment is longer. $73.39 \%$ felt that the treatment is costly. Around 52\% felt that the orthodontic treatment is longer. $73.39 \%$ felt that the treatment is costly.

Conclusion: The study's main purpose was to know the levels of awareness of orthodontic treatment need among young adults, there by educating them by posing these questions as this age group is considered to be potential orthodontic patients.
\end{abstract}

Keywords: Awareness, Survey, ANOVA.

\section{Introduction}

Health is defined by WHO as "a state of complete physical, mental, and social well-being and not merely the absence of disease or infirmity. It is influenced by various factors such as genes, lifestyle, environment, socio-economic status. Oral health is an important component of Health. It can affect the general health, social well-being, development and education. The social target of WHO is to attain a level of health that enables people to lead a socially and economically productive life. $^{1,2}$

Awareness is defined as the state or quality of being aware of something. There is a need to identify levels of awareness with respect to Orthodontic treatment need in the adult group of patients who comprise the majority of orthodontic patients. Earlier studies were conducted to assess the awareness levels of oral health and need for orthodontic treatment among pre- adolescent and adolescents. ${ }^{3-6}$ The present study was undertaken to study the levels of awareness among young adults.

\section{Materials and Methods}

A survey was conducted among the students of various colleges in Hyderabad and Secunderabad, India. A total of 808 young adult patients of age group of 1825 years were included in the study.

Ethical committee clearance was obtained from ethical committee of Army College of Dental Sciences to conduct the study and prior permissions were obtained from college management authorities.

\section{Inclusion Criteria:}

1. Students with age group of 18-25 years

2. No prior history of orthodontic treatment

3. Healthy adults

\section{Exclusion Criteria:}

1. Subjects who underwent orthodontic treatment

2. Subjects with cranio- facial anomalies like cleft lip and palate

\section{Methodology}

A pre-structured questionnaire consisting of 14 questions were given to the subjects. They were instructed to give the answers as YES/NO. Pilot study was done to validate the closed ended questionnaire. Based on the results of pilot study the sample size was decided to be 750 . The total sample composed of 808 subjects, including 491 males and 317 females.

The following questionnaire was given:

1. Have you ever noticed people with irregular teeth? a) Yes b) No

2. Have you seen people wearing braces? a) Yes b) No

3. Have you ever felt the need to wear braces? a) Yes b) No

4. Do you think crooked teeth could have ill effects if left untreated? a) Yes b) No

5. Could thumb sucking have any ill effect on front teeth alignment? a) Yes b) No

6. Do you believe teeth should be properly aligned for better facial appearance and chewing efficiency? a) Yes b) No 
7. Has anyone advised you to get your teeth aligned? a) Yes b) No

8. Have you ever visited a dentist before? a) Yes b) No

9. Do you know any specialized branch in dentistry? a) Yes b) No

10. Did you ever hear of an Orthodontist? a) Yes b) No

11. Are you aware that Orthoodntists can align your teeth? a) Yes b) No

12. Are you aware that sometimes. A few teeth may have to be removed for aligning irregular teeth? a) Yes b) No

13. Do you think the duration of Orthodontic treatment is longer than other dental procedures? a) Yes b) No

Table1: Comparison of male and females with opinion on Q. No. 1: Have you ever noticed people with irregular teeth

\begin{tabular}{|l|c|c|c|c|c|c|}
\hline Gender & Yes & \% & No & \% & Total & \% \\
\hline Male & 372 & 75.76 & 119 & 24.24 & 491 & 60.77 \\
\hline Female & 248 & 78.23 & 69 & 21.77 & 317 & 39.23 \\
\hline Total & 620 & 76.73 & 188 & 23.27 & 808 & 100.00 \\
\hline \multicolumn{7}{|c|}{ Chi-square $=0.6582$} \\
\hline
\end{tabular}

Table 2: Comparison of male and females with opinion on Q. No. 2: Have you seen people wearing braces

$* \mathrm{p}<0.05$

\begin{tabular}{|l|c|c|c|c|c|c|}
\hline Gender & Yes & \% & No & \% & Total & \% \\
\hline Male & 398 & 81.06 & 93 & 18.94 & 491 & 60.77 \\
\hline Female & 280 & 88.33 & 37 & 11.67 & 317 & 39.23 \\
\hline Total & 678 & 83.91 & 130 & 16.09 & 808 & 100.00 \\
\hline \multicolumn{7}{|c|}{ Chi-square $=7.5392$} \\
\hline \multicolumn{7}{|c|}{$\mathrm{P}=0.0061 *$} \\
\hline
\end{tabular}

Table 3: Comparison of male and females with opinion on Q. No. 3: Have you ever felt the need to wear braces

\begin{tabular}{|l|c|c|c|c|c|c|}
\hline Gender & Yes & \% & No & \% & Total & \% \\
\hline Male & 132 & 26.88 & 359 & 73.12 & 491 & 60.77 \\
\hline Female & 77 & 24.29 & 240 & 75.71 & 317 & 39.23 \\
\hline Total & 209 & 25.87 & 599 & 74.13 & 808 & 100.00 \\
\hline \multicolumn{7}{|c|}{ Chi-square $=0.6764$} \\
\hline \multicolumn{7}{|c|}{$P=0.4113$} \\
\hline
\end{tabular}

Table 4: Comparison of male and females with opinion on Q. No. 4: Do you think crooked teeth could have ill effects if left untreated

\begin{tabular}{|l|c|c|c|c|c|c|}
\hline \multicolumn{1}{|c|}{ Gender } & Yes & \% & No & \% & Total & \% \\
\hline Male & 332 & 67.62 & 159 & 32.38 & 491 & 60.77 \\
\hline Female & 218 & 68.77 & 99 & 31.23 & 317 & 39.23 \\
\hline Total & 550 & 68.07 & 258 & 31.93 & 808 & 100.00 \\
\hline \multicolumn{7}{|c|}{ Chi-square $=0.1184$ P $=0.7315$} \\
\hline
\end{tabular}

Table 5: Comparison of male and females with opinion on Q. No. 5: Could thumb sucking have any ill effects on front teeth alignment

\begin{tabular}{|l|c|c|c|c|c|c|}
\hline \multicolumn{1}{|c|}{ Gender } & Yes & \% & No & \% & Total & \% \\
\hline Male & 279 & 56.82 & 212 & 43.18 & 491 & 60.77 \\
\hline Female & 155 & 48.90 & 162 & 51.10 & 317 & 39.23 \\
\hline Total & 434 & 53.71 & 374 & 46.29 & 808 & 100.00 \\
\hline \multicolumn{6}{|c|}{ Chi-square $=4.8694$} & $\mathrm{P}=0.0273 *$ \\
\hline
\end{tabular}

$* \mathrm{p}<0.05$ 
Table 6: Comparison of male and females with opinion on Q. No. 6: Do you believe teeth should be properly aligned for better facial appearance and chewing efficiency

\begin{tabular}{|l|c|c|c|c|c|c|}
\hline Gender & Yes & \% & No & \% & Total & \% \\
\hline Male & 427 & 86.97 & 64 & 13.03 & 491 & 60.77 \\
\hline Female & 278 & 87.70 & 39 & 12.30 & 317 & 39.23 \\
\hline Total & 705 & 87.25 & 103 & 12.75 & 808 & 100.00 \\
\hline \multicolumn{7}{|c|}{ Chi-square $=0.0935 \mathrm{P}=0.7614$} \\
\hline
\end{tabular}

Table 7: Comparison of male and females with opinion on Q. No. 7: Has anyone advised you to get your teeth aligned

\begin{tabular}{|l|c|c|c|c|c|c|}
\hline Gender & Yes & \% & No & \% & Total & \% \\
\hline Male & 134 & 27.29 & 357 & 72.71 & 491 & 60.77 \\
\hline Female & 71 & 22.40 & 246 & 77.60 & 317 & 39.23 \\
\hline Total & 205 & 25.37 & 603 & 74.63 & 808 & 100.00 \\
\hline \multicolumn{7}{|c|}{ Chi-square $=2.4372$} \\
\hline \multicolumn{7}{|c|}{}
\end{tabular}

Table 8: Comparison of male and females with opinion on Q. No. 8: Have you ever visited a dentist before

\begin{tabular}{|c|c|c|c|c|c|c|}
\hline Gender & Yes & $\%$ & No & $\%$ & Total & $\%$ \\
\hline Male & 147 & 29.94 & 344 & 70.06 & 491 & 60.77 \\
\hline Female & 96 & 30.28 & 221 & 69.72 & 317 & 39.23 \\
\hline Total & 243 & 30.07 & 565 & 69.93 & 808 & 100.00 \\
\hline \multicolumn{7}{|c|}{ Chi-square $=0.0113 \mathrm{P}=0.9172$} \\
\hline
\end{tabular}

Table 9: Comparison of male and females with opinion on Q. No. 9: Do you know any specialized branch in dentistry

$* \mathrm{p}<0.05$

\begin{tabular}{|l|c|c|c|c|c|c|}
\hline Gender & Yes & \% & No & \% & Total & \% \\
\hline Male & 168 & 34.22 & 323 & 65.78 & 491 & 60.77 \\
\hline Female & 58 & 18.30 & 259 & 81.70 & 317 & 39.23 \\
\hline Total & 226 & 27.97 & 582 & 72.03 & 808 & 100.00 \\
\hline \multicolumn{8}{|c|}{ Chi-square $=24.2316 \quad \mathrm{P}=0.0001^{*}$} \\
\hline
\end{tabular}

Table 10: Comparison of male and females with opinion on Q. No. 10: In your opinion did you hear about an Orthodontist

\begin{tabular}{|l|c|c|c|c|c|c|}
\hline \multicolumn{1}{|c|}{ Gender } & Yes & \% & No & \% & Total & \% \\
\hline Male & 215 & 43.79 & 276 & 56.21 & 491 & 60.77 \\
\hline Female & 154 & 48.58 & 163 & 51.42 & 317 & 39.23 \\
\hline Total & 369 & 45.67 & 439 & 54.33 & 808 & 100.00 \\
\hline \multicolumn{7}{|c|}{ Chi-square $=1.7833$} \\
\hline
\end{tabular}

Table 11: Comparison of male and females with opinion on Q. No. 11: Are you aware that orthodontist can align your teeth

$* \mathrm{p}<0.05$

\begin{tabular}{|l|c|c|c|c|c|c|}
\hline Gender & Yes & \% & No & \% & Total & \% \\
\hline Male & 276 & 56.21 & 215 & 43.79 & 491 & 60.77 \\
\hline Female & 154 & 48.58 & 163 & 51.42 & 317 & 39.23 \\
\hline Total & 430 & 53.22 & 378 & 46.78 & 808 & 100.00 \\
\hline \multicolumn{6}{|c|}{ Chi-square $=4.5068 \mathrm{P}=0.0347 *$} \\
\hline
\end{tabular}

Table 12: Comparison of male and females with opinion on Q. No. 12: Are you aware that sometimes, a few teeth may sometimes have to be removed for aligning irregular teeth

\begin{tabular}{|l|c|c|c|c|c|c|}
\hline \multicolumn{1}{|c|}{ Gender } & Yes & \% & No & \% & Total & \% \\
\hline Male & 312 & 59.32 & 214 & 40.68 & 526 & 65.10 \\
\hline Female & 179 & 63.48 & 103 & 36.52 & 282 & 34.90 \\
\hline Total & 491 & 60.77 & 317 & 39.23 & 808 & 100.00 \\
\hline \multicolumn{7}{|c|}{ Chi-square $=1.3322$} \\
\hline
\end{tabular}


Table 13: Comparison of male and females with opinion on Q. No. 13: Do you think duration of orthodontic treatment is longer than other dental procedures

\begin{tabular}{|l|c|c|c|c|c|c|}
\hline Gender & Yes & \% & No & \% & Total & \% \\
\hline Male & 251 & 51.12 & 240 & 48.88 & 491 & 60.77 \\
\hline Female & 163 & 51.42 & 154 & 48.58 & 317 & 39.23 \\
\hline Total & 414 & 51.24 & 394 & 48.76 & 808 & 100.00 \\
\hline \multicolumn{7}{|c|}{ Chi-square $=0.0073$} \\
\hline \multicolumn{7}{|c|}{$\mathrm{P}=0.9342$} \\
\hline
\end{tabular}

Table 14: Comparison of male and females with opinion on Q. No. 13: Do you think orthodontic treatment is costly?

\begin{tabular}{|l|c|c|c|c|c|c|}
\hline \multicolumn{1}{|c|}{ Gender } & Yes & \% & No & \% & Total & \% \\
\hline Male & 357 & 72.71 & 134 & 27.29 & 491 & 60.77 \\
\hline Female & 236 & 74.45 & 81 & 25.55 & 317 & 39.23 \\
\hline Total & 593 & 73.39 & 215 & 26.61 & 808 & 100.00 \\
\hline \multicolumn{7}{|c|}{ Chi-square $=0.2983$} \\
\hline \multicolumn{7}{|c|}{ P $=0.5852$} \\
\hline
\end{tabular}

Table 15: Male and females comparison in each question

\begin{tabular}{|c|c|c|c|c|c|c|c|}
\hline Questions & Gender & Yes & $\%$ & No & $\%$ & Total & $\%$ \\
\hline \multirow{3}{*}{$\begin{array}{l}\text { 1. Have you ever noticed people with } \\
\text { irregular teeth }\end{array}$} & Male & 372 & 75.76 & 119 & 24.24 & 491 & 60.77 \\
\hline & Female & 248 & 78.23 & 69 & 21.77 & 317 & 39.23 \\
\hline & Total & 620 & 76.73 & 188 & 23.27 & 808 & 100.00 \\
\hline \multirow[t]{3}{*}{ 2.Have you seen people wearing braces } & Male & 398 & 81.06 & 93 & 18.94 & 491 & 60.77 \\
\hline & Female & 280 & 88.33 & 37 & 11.67 & 317 & 39.23 \\
\hline & Total & 678 & 83.91 & 130 & 16.09 & 808 & 100.00 \\
\hline \multirow{3}{*}{$\begin{array}{l}\text { 3. Have you ever felt the need to wear } \\
\text { braces }\end{array}$} & Male & 132 & 26.88 & 359 & 73.12 & 491 & 60.77 \\
\hline & Female & 77 & 24.29 & 240 & 75.71 & 317 & 39.23 \\
\hline & Total & 209 & 25.87 & 599 & 74.13 & 808 & 100.00 \\
\hline \multirow{3}{*}{$\begin{array}{l}\text { 4. Do you think crooked teeth could } \\
\text { have ill effects if left untreated }\end{array}$} & Male & 332 & 67.62 & 159 & 32.38 & 491 & 60.77 \\
\hline & Female & 218 & 68.77 & 99 & 31.23 & 317 & 39.23 \\
\hline & Total & 550 & 68.07 & 258 & 31.93 & 808 & 100.00 \\
\hline \multirow{3}{*}{$\begin{array}{l}\text { 5. Could thumb sucking have any ill } \\
\text { effects on front teeth alignment }\end{array}$} & Male & 279 & 56.82 & 212 & 43.18 & 491 & 60.77 \\
\hline & Female & 155 & 48.90 & 162 & 51.10 & 317 & 39.23 \\
\hline & Total & 434 & 53.71 & 374 & 46.29 & 808 & 100.00 \\
\hline \multirow{3}{*}{$\begin{array}{l}\text { 6. Do you believe teeth should be } \\
\text { properly ligned for better facial } \\
\text { appearance and hewing efficiency }\end{array}$} & Male & 427 & 86.97 & 64 & 13.03 & 491 & 60.77 \\
\hline & Female & 278 & 87.70 & 39 & 12.30 & 317 & 39.23 \\
\hline & Total & 705 & 87.25 & 103 & 12.75 & 808 & 100.00 \\
\hline \multirow{3}{*}{$\begin{array}{l}\text { 7. Has anyone advised you to get your } \\
\text { teeth aligned }\end{array}$} & Male & 134 & 27.29 & 357 & 72.71 & 491 & 60.77 \\
\hline & Female & 71 & 22.40 & 246 & 77.60 & 317 & 39.23 \\
\hline & Total & 205 & 25.37 & 603 & 74.63 & 808 & 100.00 \\
\hline \multirow{3}{*}{$\begin{array}{l}\text { 8. Have you ever visited a dentist } \\
\text { before }\end{array}$} & Male & 147 & 29.94 & 344 & 70.06 & 491 & 60.77 \\
\hline & Female & 96 & 30.28 & 221 & 69.72 & 317 & 39.23 \\
\hline & Total & 243 & 30.07 & 565 & 69.93 & 808 & 100.00 \\
\hline \multirow{3}{*}{$\begin{array}{l}\text { 9. Do you know any specialized branch } \\
\text { in dentistry }\end{array}$} & Male & 168 & 34.22 & 323 & 65.78 & 491 & 60.77 \\
\hline & Female & 58 & 18.30 & 259 & 81.70 & 317 & 39.23 \\
\hline & Total & 226 & 27.97 & 582 & 72.03 & 808 & 100.00 \\
\hline \multirow{3}{*}{$\begin{array}{l}\text { 10. In your opinion did you hear about } \\
\text { an Orthodontist }\end{array}$} & Male & 215 & 43.79 & 276 & 56.21 & 491 & 60.77 \\
\hline & Female & 154 & 48.58 & 163 & 51.42 & 317 & 39.23 \\
\hline & Total & 369 & 45.67 & 439 & 54.33 & 808 & 100.00 \\
\hline \multirow{3}{*}{$\begin{array}{l}\text { 11.Are you aware that orthodontist can } \\
\text { align your teeth }\end{array}$} & Male & 276 & 56.21 & 215 & 43.79 & 491 & 60.77 \\
\hline & Female & 154 & 48.58 & 163 & 51.42 & 317 & 39.23 \\
\hline & Total & 430 & 53.22 & 378 & 46.78 & 808 & 100.00 \\
\hline \multirow{3}{*}{$\begin{array}{l}\text { 12. Are you aware that sometimes, a } \\
\text { few teeth may sometimes have to be } \\
\text { removed for aligning irregular teeth }\end{array}$} & Male & 312 & 59.32 & 214 & 40.68 & 526 & 65.10 \\
\hline & Female & 179 & 63.48 & 103 & 36.52 & 282 & 34.90 \\
\hline & Total & 491 & 60.77 & 317 & 39.23 & 808 & 100.00 \\
\hline
\end{tabular}




\begin{tabular}{|l|c|c|c|c|c|c|c|}
\hline \multirow{2}{*}{$\begin{array}{l}\text { 13. Do you think duration of orthodontic } \\
\text { treatment is longer than other dental } \\
\text { procedures }\end{array}$} & Male & 251 & 51.12 & 240 & 48.88 & 491 & 60.77 \\
\cline { 2 - 8 } & Female & 163 & 51.42 & 154 & 48.58 & 317 & 39.23 \\
\cline { 2 - 8 } & Total & 414 & 51.24 & 394 & 48.76 & 808 & 100.00 \\
\hline \multirow{2}{*}{$\begin{array}{l}\text { 14. Do you think orthodontic treatment } \\
\text { is costly? }\end{array}$} & Male & 357 & 72.71 & 134 & 27.29 & 491 & 60.77 \\
\cline { 2 - 8 } & Female & 236 & 74.45 & 81 & 25.55 & 317 & 39.23 \\
\cline { 2 - 8 } & Total & 593 & 73.39 & 215 & 26.61 & 808 & 100.00 \\
\hline
\end{tabular}

\section{Discussion}

The study was designed to determine awareness of orthodontic treatment need among young adults. A questionnaire was given to 808 in different educational institutions. Most of the students participated in the survey responded that they noticed people with crooked teeth and had seen people wearing braces $(76.73 \%$ and $83.91 \%$ respectively). However, when asked the need for wearing braces, most of them responded as not required $(74.13 \%)$. Majority of the students responded on a positive note when questioned about functional ill effects with malocclusion showing that awareness among young adults is good about oral health. On the contrast when questioned about visiting a dentist majority of them have not visited a dentist and had no knowledge about specializations in dentistry $(69.93 \%$ and $72.03 \%$ ). Through the survey it is inferred that 54\% of students were unaware of orthodontist and 46\% claim to know who an Orthodontist is. Majority responded that in few cases teeth will have to be removed for aligning the irregular teeth. (60.77\%). Around $52 \%$ felt that the orthodontic treatment is longer. $73.39 \%$ felt that the treatment is costly.

Earlier studies were done by Roopa Siddegowda and MS Rani ${ }^{2,45}$ among school children based on origin and different socio-economic backgrounds and concluded that the awareness among school children was average and there was a need to educate children on oral health and malocclusion related problems. The present study is unique in its kind as the subjects taken are young adults who are supposed to be potential orthodontic patients.

\section{Conclusion}

The study's main purpose was to know the levels of awareness of orthodontic treatment need among young adults, there by educating them by posing these questions as this age group is considered to be potential orthodontic patients. The survey will be useful to the community, dental professionals to provide the students with awareness programs and treatment possibilities.
3. Muqtadir Quadri SM, Thilagarani PR et al. Awareness towards orthodontic treatment in central Indian school children. Sch. J. Dent. Sci.,2015;2(1):45-48.

4. Siddegowda R, Rani MS. An epidemiological survey on awareness towards Orthodontic treatment in South Indian school children. Open Journal of Dentistry and Oral medicine. 2013;1(1):5-8.

5. Siddegowda R, Rani MS. Awareness of Orthodontic Treatment in school children of Karnataka state- A survey. Journal of Applied Physics.2013;4(2):1-5.

6. Rafighi A, Moghaddam SF. Awareness of Orthodontic treatments among school teachers of two cities of Iran. $J$ Dent Res Clin Dent Prospect 2012;6(1):25-28.

\section{References}

1. Mahajan BK. Social Environment. Text book of Preventive and Social Medicine. $1^{\text {st }}$ Ed. New Delhi, Jaypee Brothers Medical Publishers (P) ltd;29-35.

2. Siddegowda R. Evaluating the awareness of Orthodontic treatment mong school children of different Socioeconomic grounds. International Journal of Scientific Research. 2013;2(7):370-371. 